\title{
Uncertainties of a Linear Variable Differential Inductor Probe for Picometer Resolution Measurement Systems
}

Aschenbrenner, Bernhard; Zagar Bernhard

Institute for Measurement Technology / Johannes Kepler University Linz

Altenbergerstraße 69, A - 4040 Linz

ABSTRACT - Linear Variable Differential Inductor (LVDI) probes are used for most precise roundness and roughness measurements down to sub-nanometer. Even under rough conditions the LVDI guarantees a good repeatability of the measurement. In this paper some widely overlooked sources of errors that-if unavoidable-increase measurement uncertainty beyond the theoretical limit attainable are discussed and the magnitude of their influence is detailed. Usually safely ignored influences like stochastic noise, heating and elastic deformation can result in uncertainties of several nanometers.

\section{Introduction}

In the paper a range of parameters are discussed that can potentially lead to uncertainties in a highest resolution displacement measurement system. This system is able to resolve - by averaging over several measurements - in the sub-nanometer range. The ever increasing quality demand of machine components and machines in industry has been the driving force behind research for more accurate measurement methods. Precision measurement systems that can achieve a displacement accuracy in the order of nanometers are essential in the optical, semiconductor and nanotechnology industry and ultra-precise machining. Capacitive sensors and linear variable differential inductor (LVDI) can measure displacements down to the sub-nanometer range [1] (see Figure 1). Alternatively, laser interferometers may be used for measuring displacements with nanometer accuracy. However, those incurs high costs, require relatively large space for installation and operation and demand for very well controlled environmental conditions [2].

In analyzing sensors and system behavior for very high resolution systems the number of parameters that are influencing accuracy and resolution rapidly grows beyond the typical parameters like temperature, noise, quantization effects, to name just a few. In systems that strive for sub nm-resolution even usually safely ignored influences like static friction, dynamic friction, heating, elastic deformation, and many more can result in uncertainties of several nanometers. Pretty much every conceivable, environmental parameter needs to be analyzed for its possible influence. The purpose of this research is to clarify the influences of some of the parameters in ultra-precision measurement.
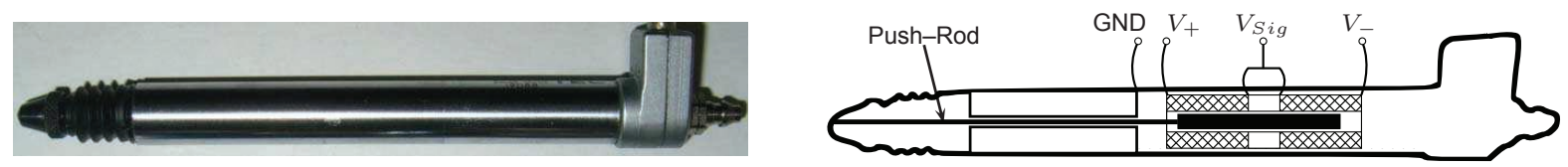

Fig. 1: The used LVDI and its principle sketch. In the sketch can be seen the coil with the center tab and the push rod. The differential $5 \mathrm{~V}$ peak to peak voltage is applied to the connectors $V_{+}$and $V_{-}$.

\section{Thermal Noise Behavior of the Bridge Circuit}

In this section the noise voltages which are generated from the resistive bridge components and disturbs the diagonal voltage $u_{d}$ will be calculated. The resistive components are the $R_{D C}$ of the sensor, $R_{B}$ and $R_{S}$ whose noise significantly influence the diagonal voltage $u_{d}$ of the reactive deflection bridge, thus the measurement accuracy. The LVDI is a branch of a reactive deflection bridge which is supplied with two differential sinusoidal voltages (see Figure 2). The resistor noise is well modeled by a Gaussian white noise.

Its one-sided Power Density Spectrum (PSD) for a voltage and current source is given by:

$$
S_{n, v}(\omega)=\frac{2}{\pi} k T R, \quad 0 \leq \omega \leq B \quad \text { and } \quad S_{n, i}(\omega)=\frac{2 k T}{\pi R}, \quad 0 \leq \omega \leq B
$$

where $k$ is the Boltzmann constant $\left(k=1.38 \times 10^{-23} \frac{\mathrm{Ws}}{\mathrm{K}}\right), T$ the absolute temperature in Kelvin of the components $(T=300 \mathrm{~K}), B$ the bandwidth in rad/s and $R$ the ohmic resistance in ohm. The noise sources are statistically independent, the power spectral density of the root mean square (r.m.s) current $I_{n, R_{S}}$ is 


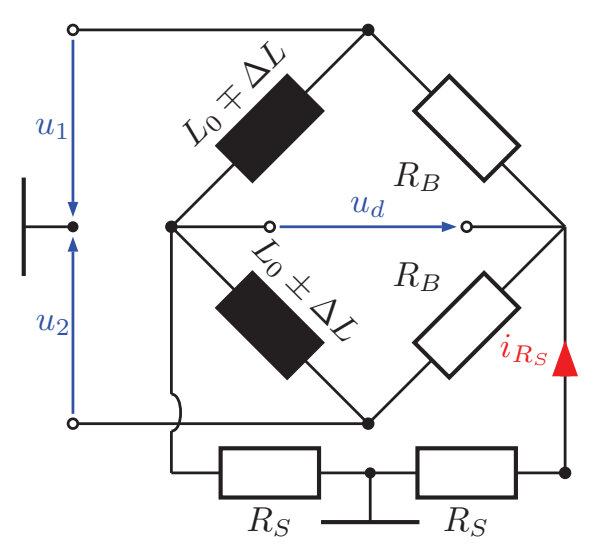

Fig. 2: The linear noise-free reactive deflection bridge for an inductive push-pull displacement sensor. The two resistors labelled $R_{S}$ are necessary, according to the data sheet, to obtain the sensitivity of $76 \frac{\mathrm{V}}{\mathrm{Vm}}$.

simply the sum of the power spectral densities of the noise currents. In a given bandwidth $B$ the total r.m.s current noise passing trough the resistor $R_{S}^{\prime}=2 R_{S}$ can be calculated by:

$$
I_{n, R_{S}}=\sqrt{\int_{\omega_{1}}^{\omega_{2}} \sum_{i=1}^{N} S_{n, i} \mathrm{~d} \omega}
$$

where $\omega_{1}=2 \pi f_{1}$ and $\omega_{2}=2 \pi f_{2}$ the lower and upper angular frequencies of the passband. The electrical parameters of the Sensor are $C=55 \mathrm{pF}, L=1.8 \mathrm{mH}$ and $R_{D C}=332 \Omega$. These parameters were measured by an HP 4194A impedance gain phase analyzer. The other bridge parameters are $R_{B}=680 \Omega$ and $R_{S}=6800 \Omega$. The frequency $f_{2}=160 \mathrm{kHz}$ is the $-3 \mathrm{~dB}$ frequency of a first order low pass, and has to be multiplied with a correction factor $\pi / 2$ to obtain the equivalent noise bandwidth for a first order low pass filter. For a given bandwidth of $B=160 \mathrm{kHz}$, the noise voltage is $U_{n, d}=I_{n, R s} 2 R_{S}=1.29 \mu \mathrm{V}$.

The bridge has a differential, $16 \mathrm{kHz}, u_{1}=u_{2}=5 \mathrm{~V}$ peak-to-peak sinusoidal voltage supply and the sensor has a sensitivity of $76 \frac{\mathrm{V}}{\mathrm{Vm}}$ with a resistance $R_{S}=6.8 \mathrm{k} \Omega$ at its output (see Figure 2). Hence, the noise voltage $U_{n, d}$ contributes to a measurement uncertainty of $\sigma_{e, n}= \pm 2.4 \mathrm{~nm}$.

\section{Thermal Expansion}

\section{A. Thermal expansion due to Handling}

Handling of the specimen of the measurement system influences the measurement system by the thermal energy introduced and can affect the measurement result significantly. The operating person touching the sensor or the measurement system will introduce thermal energy causing the measurement system to heat up. Certainly, the exchange of energy will be very small but the effect to the measurement system can be significantly.

To be able to estimate these influences an experiment was performed using an aluminium cylinder as model of the mechanical stage. A Pt-100 RTD was attached (see Figure 3) and the temperature increase caused by a mere touching for $30 \mathrm{~s}$ with one hand was recorded. Based on a temperature of $20^{\circ} \mathrm{C}$ the increase $T_{T, c y l}=0.54^{\circ} \mathrm{C}$ was determined and used to estimate any thermally induced changes in measurement geometry. The thermal expansion coefficient of aluminium is assumed to be $\alpha_{a l}=23 \cdot 10^{-6} / \mathrm{K}$ and the linear dimension of the mechanics is assumed to be $250 \mathrm{~mm}$ resulting in a transient expansion of $\Delta h_{T, c y l}=$ $7.44 \mu \mathrm{m}$ of which only the transient expansion during the measurement cycle (60 s max.) will influence the measurand.

This simple example shows that the thermal energy from an operating person can greatly influence the measurement system and cause a transient expansion with magnitudes that are larger than commonly thought.

\section{B. Thermal Expansion due to Power Dissipation}

As is be illustrated in Figure 1 a typical LVDI consists of a ferritic steel tube, push/pull rod, armature and sensor coil with center tab. The power dissipation of the sensor contributes to a thermal expansion of all 
mechanical components [3]. The sensor coil is current-carrying and losses occur. The power dissipation increases over time the temperature.Therefore, a thermal expansion occurs in the heated components. This section shows a simple analytical approximation of the amount of thermal expansion uncertainties compared to the results of a long term stability test. By using the first law of thermodynamics, the energy balance of the sensor is

$$
P_{L} \Delta t=\alpha_{C u} S \Delta \vartheta \Delta t+c_{C u} m \Delta \vartheta
$$

The solution of Equation 3 with respect to time $t$ provides the temperature profile of the inductor and its armature. The solution is given by the exponential function:

$$
\Delta \vartheta(t)=\Delta \vartheta_{\text {end }}\left(1-\exp ^{-\frac{t}{\tau}}\right)
$$

where $\Delta \vartheta_{\text {end }}=P_{L} /\left(\alpha_{C u} \cdot S\right)$ is the temperature increase of the sensor coil and armature and $\tau=c_{C u}$. $\mathrm{m} /\left(\alpha_{C u} \cdot S\right)$ is the time constant. Furthermore, the variables $\alpha_{C u}$ is the heat transfer coefficient of copper $\left(\alpha_{C u}=12 \mathrm{~W} /\left(\mathrm{m}^{2} \cdot \mathrm{K}\right)\right), S$ the surface of the heat emitting sensor part, $c_{C u}$ the specific heat capacity of copper $\left(c_{C u}=385 \mathrm{Ws} /(\mathrm{kg} \cdot \mathrm{K})\right), m$ the mass and $\Delta \vartheta(t)$ the temperature difference.

For a power dissipation of $P_{S, l o s s}=58 \mathrm{~mW}$ a coil mass $m=57 \mathrm{~g}$ and a surface $S=520 \mathrm{~mm}^{2}$ given a thermal expansion coefficient of stainless steel $\alpha_{F e}=11 \cdot 10^{-6} / \mathrm{K}$ and a length of the armature $l_{A}=25 \mathrm{~mm}$ a warm-up behavior as shown in Figure 4 has been determined. After a warming-up phase of $1800 \mathrm{~s}$ the sensor is in the thermal equilibrium but still exhibits large random components of its expansion that are on the order of $5-10 \mathrm{~nm}$ over a period of $100 \mathrm{~s}$.

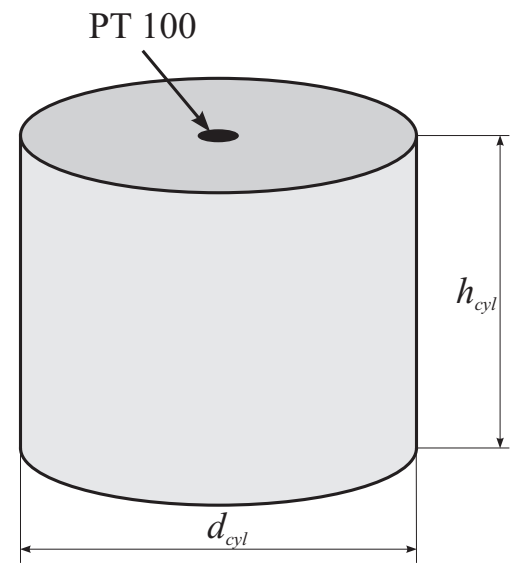

Fig. 3: The cylinder for the handling simulation having a diameter of $d_{c y l}=80 \mathrm{~mm}$, a height of $h_{c y l}=80 \mathrm{~mm}$ and a mass of $m_{c y l}=1.1 \mathrm{~kg}$.

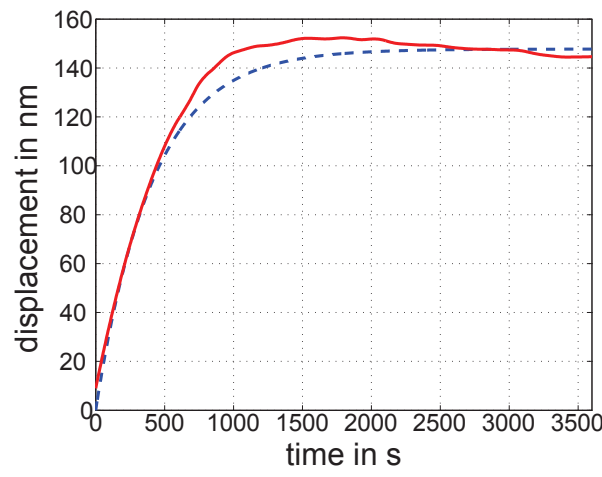

Fig. 4: A long term stability test with a typical drift as seen in a precise measurement system. The blue dashed line is the simulated curve and the red solid line represents the experimental results.

\section{Elastic Deformation}

Inevitable elastic deformations occur where a mechanical force is exerted on a specimen through a tactile sensor such as an LVDI probe. These probes which are used for precise displacement, roundness and roughness measurements react upon the specimen and the mechanical components of the probe such as the push rod and the ball tip yield due to the usually small measurement force. Even contact forces as low as $F_{\text {cont }}=0.1 \mathrm{~N}$ contribute significantly to the measurement uncertainty as will be shown. The better the resolution of the system becomes the more the influence of the contact forces increase. The measuring or contact force at zero displacement applied to the specimen due to the pre-stressed return spring is (dependent on the measurand and displaying a randomly varying component) between $0.45 \mathrm{~N}$ $\leq F_{\text {cont }} \leq 0.75 \mathrm{~N}$. To analyze this problem thoroughly the force caused by the return spring stiffness $c_{R S}$ and including the bearing friction is obtained by measuring the probe contact force at different probes displacements. A sensitive scale measured the contact force by moving the sensor stepwise (in $100 \mu \mathrm{m}$ increments) to measurement positions between $-1.2 \mathrm{~mm}$ to $1.5 \mathrm{~mm}$ about the zero point. In Figure 5 the measurements are displayed and the spring stiffness $c_{R S}$ is obtained by determining the linear trend. The same spring stiffness value $c_{R S}=190 \mathrm{~N} / \mathrm{m}$ is obtained for ascending (red line) and descending (blue line) displacements. The varying component as measured by its standard deviation $\sigma_{m, R S}= \pm 0.046 \mathrm{~N}$. 


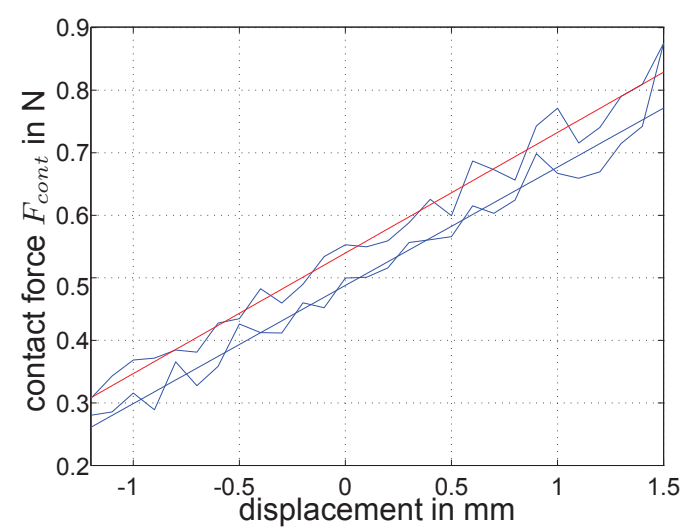

Fig. 5: The probe contact force applied to the specimen due to the return spring vs. displacement.

\section{A. Push-Rod Compression}

As is visualized in Fig. 6 the mechanical arrangement of the push rod can be thought of as the return spring in series with the stiffness of the push rod, where the measurand is taken at the mid-point position. To estimate the influence on the measurand of the small but non-negligible random force component that compresses the push rod the following is assumed. The static force is given for the mid-point position. The pre-stressed return spring then exerts a force of approximately $F_{\text {cont }}=0.45 \mathrm{~N}$. The stainless steel push rod is $l_{0}=20 \mathrm{~mm}$ long, has a cross section of $A_{p}=12.4 \mathrm{~mm}^{2}$ and a Youngs's modulus of $E_{p}=210 \mathrm{GPa}$. The axial static measurement force contributes to a $\Delta X_{m, P R}=3.5 \mathrm{~nm}$ static compression of the push rod at the operating point. The uncertainty of this force according to the section above leads to an additional mechanical random compression of $\sigma_{m, P R}= \pm 312 \mathrm{pm}$.

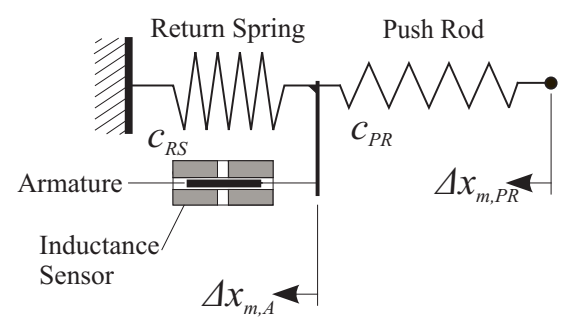

Fig. 6: A simple mechanically equivalent system of the sensor with its return spring and its push rod which has been replaced by a coil spring with the stiffness $c_{P R}$. The variables $\Delta x_{m, A}$ and $\Delta x_{m, P R}$ are the armature and push-rod displacements.

The systematic error due to the sensor deflection can be calculated by

$$
\Delta x_{m, A}=\left(1-\frac{c_{P R}}{c_{P R}+c_{R S}}\right) \Delta x_{m, P R} .
$$

where $c_{P R}=E_{P R} \cdot A_{P R} / l_{0}=130 \mathrm{MN} / \mathrm{m}$ is the stiffness of the push rod, $c_{R S}=190 \mathrm{~N} / \mathrm{m}$ is the stiffness of the return spring and the $\Delta x_{m, P R}$ the mechanical displacement of the push rod. The systematic error is the difference of $\Delta x_{m, P R}=\Delta x_{m, P R}-\Delta x_{m, A}$ (see Figure 6). A displacement of $\Delta x_{m, P R}=1 \mathrm{~nm}$ contributes to a systematic error of $\Delta x_{m, A}=1.5 \mathrm{fm}$. The error rise with the displacement and at maximum measurement range $\left(\Delta x_{m, P R}= \pm 50 \mu \mathrm{m}\right)$ the systematic error is $\Delta x_{m, A}=73 \mathrm{pm}$. This error does not affect the measurement result significantly, due to it being static.

\section{B. Elastic Deformation of the Specimen}

The ruby ball tip of the probe react upon the specimen. The small contact surface contributes to large pressure despite the small forces acting. For the calculation of the elastic deformation, the specimen is assumed flat or the specimen radius is considerably larger than the ruby ball radius $R=2.5 \mathrm{~mm}$. Thus, the specimen can be considered as an elastic half-space (see Figure 7). The mechanical displacement $\Delta x_{m, S}$ is related to the applied force $F_{\text {cont }}$ by [7]: 


$$
x_{m, S}=\sqrt[3]{\left(\frac{3}{4} \frac{F_{\text {cont }}}{E^{\prime} \sqrt{R}}\right)^{2}} \quad \text { with } \quad E^{\prime}=\frac{E_{R} E_{S}}{E_{R}+E_{S}-E_{S} \nu_{R}^{2}-E_{R} \nu_{S}^{2}}
$$

and $E_{S}, E_{R}$ are the Young's modulus and $\nu_{S}, \nu_{R}$ the Poisson's ratios of the specimen and the ruby ball tip, respectively. The measuring contact force at zero point $\left(F_{\text {cont }}=0.45 \mathrm{~N}\right)$ contributes to a displacement of $\Delta x_{m, S}=163 \mathrm{~nm}$. The Young's modulus and Poisson's ratio for the ruby are $E_{R}=350 \mathrm{GPa}$ and $\nu_{R}=$ 0.315 and for the steel specimen $E_{S}=210 \mathrm{GPa}$ and $\nu_{S}=0.3$. Movements about the operating point also cause additional elastic deformations and displacements $\Delta x_{m, S}\left(\Delta F_{\text {cont }}\right)=\Delta x_{m, S}\left(F_{\text {cont }}+\Delta F_{\text {cont }}\right)-$ $\Delta x_{m, S}\left(\Delta F_{\text {cont }}\right)$ in dependence on the moving direction. Because of the finite specimen stiffness a certain amount of deformation occurs. This error rises with the displacement and at maximum measurement range $\left(\Delta x_{m, P R}= \pm 50 \mu \mathrm{m}\right)$ the systematic error of the specimen deformation is $\Delta x_{m, S}\left(\Delta F_{\text {cont }}\right)= \pm 2.3 \mathrm{~nm}$.

\section{Errors due to Bearing Play}

If the specimen is in motion the probe tip will be deflected into the motion direction (see Figure 8). The tangential friction force between the probe and specimen surface causes this deflection. Furthermore, the bearing play and the finite push rod bending stiffness are responsible for the tangential deflection distance (y-direction) of the push rod.

In this subsection will be discussed the error due to the bearing play, which admits a toppling of the push rod. In the next subsection the error due to the bending is discussed.

Every bearing has a clearance even very precisely manufactured ones. In this case the clearance in radial direction causes measurement uncertainties. The axis direction or $x$-direction do not affect the measurement result because of the pre-stressed return spring. The measurement uncertainty can easily be calculated by

$$
\Delta x_{m, B}=l_{0}\left(1-\cos \arcsin \frac{\Delta y}{l_{0}}\right)
$$

where $\Delta y$ is the defletion in $y$-direction of the push rod tip and $l_{0}$ the length of the push rod between the ball tip and the center among the two push rod bearings.

A $\Delta y=1 \mu \mathrm{m}$ deflection and a length $l_{0}=29 \mathrm{~mm}$ (see Figure 8) causes a error of $\Delta x_{m, B}=17 \mathrm{pm}$ in $x$-direction.

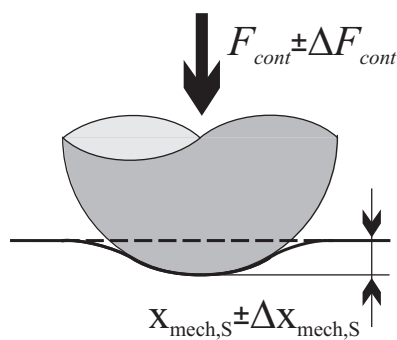

Fig. 7: An elastic sphere of radius $R$ indents an elastic half-space to depth $x_{m, s}$

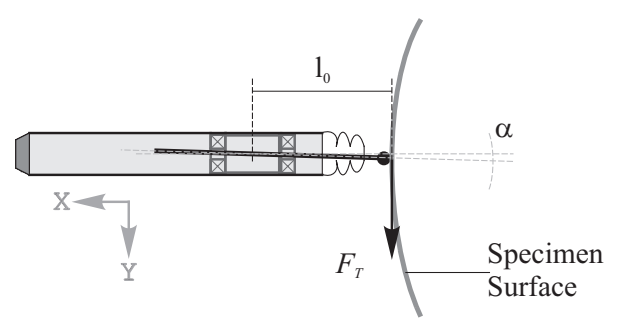

Fig. 8: Push rod toppling due to the bearing play and the tangential force $F_{T}$ causes a push rod deflection in $y$-direction.

\section{Error due to Push Rod Flex}

As mentioned in the subsection above due to the tangential force $F_{T}$ (see Figure 8 ) the push rod has a curved shape and is not completely straight. Therefore, an error occurs in $x$-direction. In Figure 9 is illustrated the qualitative push rod behavior which is caused by the force $F_{T}$.

As an example a very low tangential force $F_{T}=0.1 \mathrm{~N}$ causes a deflection of $y_{C}=100 \mathrm{~nm}$ at the push rod tip C [6]. This result can be calculated with

$$
\Delta y_{C}=\frac{F_{T} l_{a}^{3}}{E I} \frac{l_{a}^{2}}{a^{2}}\left(1+\frac{l_{a}}{a}\right) \frac{1}{3}
$$


where $a=26.5 \mathrm{~mm}$ is the distance between the two push rod bearings and $l_{a}=14 \mathrm{~mm}$ the probe zero point distance between the push rod top and the bearing $B$ (see Figure 9)[6]. The measurement uncertainty due to the push rod flex $\Delta x_{m, F}$ can be calculated by

$$
\Delta x_{m, F_{T}}=l_{0}\left(1-\cos \arcsin \frac{\Delta y_{C}}{l_{a}}\right) .
$$

A $y_{C}=100 \mathrm{~nm}$ deflection causes an error of $0.4 \mathrm{pm}$ in $x$-direction. Hence, this influence is very small and does not influence significantly the precision.

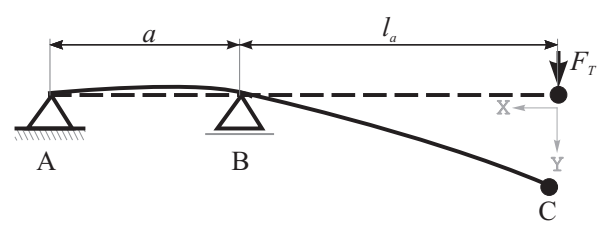

Fig. 9: Probe push rod shown as torsion free cantilever and a single force $F_{T}$.

\section{Summary and Conclusion}

In this contribution some easily overlooked error sources in sub-nanometer measurement systems were discussed and it was shown that each error term can in the adverse case be orders of magnitudes larger than the typical resolution a user assumes from these systems. In section II. the thermal noise can achieve an higher amount than the signal amplitude and yield to an poor SNR and contributes to an measurement uncertainty of $\operatorname{sigma}_{e, n}= \pm 2.4 \mathrm{~nm}$. However, SNR improvement can be done by averaging. section III. considers the influence of the handling and power dissipation. A simple experiment showed that the thermal energy from an operating person can cause a transient expansion with magnitudes larger than thought. In section IV. were static and systematic errors discussed due to the sensor contact force. Furthermore, the systematic push rod error is $\Delta x_{m} A=73 \mathrm{pm}$ and the specimen error is $\Delta x_{m, S}= \pm 2.3 \mathrm{~nm}$ due to the deformation. Additional, a random force $\sigma_{m, R S}=0.46 \mathrm{~N}$ due to the return spring and bering friction adds a measurement uncertainty (mechanical random push rod compression $\sigma_{m, P R}=312 \mathrm{pm}$ ) to the systematic errors.

\section{Acknowledgment}

The authors acknowledge the partial financial support for the work presented in this paper by the Austrian Center of Competence in Mechatronics (ACCM) in close collaboration with the Quality Technology Centre (QTC) of SKF Österreich AG.

[1] J. Sieh, J.E. Huber, N.A. Fleck, M.F. Ashby, The Selection of Sensors, Prog. Mater. Sci. 46, 2001.

[2] M. Kim, W. Moon, E.Yoon, K.R. Lee, A New Capacitve Displacement Sensor With High Accuracy And Long Range, www.sciencedirect.com, Seoul, 2006.

[3] Bernhard G. Zagar, A Discussion of Sources of Error in Laser-Speckle Based Systems,Proceedings of the 19th IMEKO TC2, 93-97,2010

[4] G.J. Coram, B.D.O. Anderson, and J.L. Wyatt, Jr., Thermal Noise Behavior of the Bridge Circuit, RLE Technical Memo, MIT Libaries, 2000.

[5] R. Fischer, Elektrische Maschinen, 11. korrigierte Auflage, München Wien, Hanser, 2001, ISBN 3-44621810-6.

[6] Horst Czichos, Hütte, 31. neubearbeitete und erweiterte Auflage, Berlin, Springer, 1990, ISBN 3-54066882-9.

[7] W. Beitz, K.-H. Grote,Dubbel - Taschenbuch für den Maschinenbau, Berlin, Springer, ISBN 3-54052381-2. 